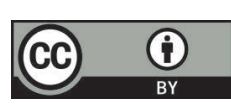

\title{
Facing up to COVID-19: role of the Epidemiology Unit in surveillance during the outbreak response in Sri Lanka
}

\section{Nimali Widanapathirana ${ }^{1 *}$, Deepa Gamage ${ }^{1}$, Chandima Hemachandra ${ }^{1}$, Thilanga Ruwanpathirana ${ }^{1}$, Chintha Jayasinghe ${ }^{1}$, Manjula Kariyawasam ${ }^{1}$, Sashimali Wickramasinghe ${ }^{1}$, Alinda Perera ${ }^{1}$, Thiraj Haputhanthri ${ }^{1}$, Sudath Samaraweera ${ }^{1}$, Paba Palihawadana ${ }^{2}$, Samitha Ginige ${ }^{1}$}

${ }^{1}$ Epidemiology Unit, Ministry of Health, Sri Lanka; ${ }^{2}$ Office of the Deputy Director General (Public Health Services) 1, Ministry of Health, Sri Lanka

"Correspondence: nimalidw@gmail.com

iDhttps://orcid.org/0000-0003-0652-5062

DOI: https://doi.org/10.4038/jccpsl.v26i5.8357

Received on 20 May 2020

Accepted on 25 May 2020

\section{Highlights}

- Epidemiological surveillance is key to an effective pandemic response.

- Preventing community transmission was challenging amidst the influx of returnees from abroad. The robust surveillance for COVID-19 adopted in the backdrop of a well-established communicable disease surveillance system was pivotal to contain the virus at the source.

\section{Background}

The first imported cases of the novel coronavirus, which originated in Wuhan, China in December 2019 were reported from Thailand and Japan in midJanuary 2020, signalling the spread of infection beyond China. Europe and the Region of Americas reported their first cases towards the end of January (1). The virus had spread in such epidemic proportions that the World Health Organization (WHO) declared the outbreak a 'Public Health Emergency of International Concern' on 30 January 2020(2). Countries were called to action to curtail the spread of a virus that was novel, with its transmission dynamics hitherto unknown. By the time this declaration was made, Sri Lanka had reported its first imported case from Wuhan, and the country was well on its way to respond to the emerging threat. The Epidemiology Unit, which is the National Focal Point for Communicable Disease Control and Prevention took swift action to face the impending epidemic of COVID-19 in the country. This report outlines the key actions taken during preparedness and response to COVID-19 in Sri Lanka by the Epidemiology Unit. The guiding principles were to contain the spread of the disease, minimize deaths due to COVID-19 and achieve a level of control with minimal or no transmission without overwhelming the health system of the country. The response has been adaptive, following the trajectory of the epidemic 
curve, intensifying case detection, contact tracing and isolation augmented, with a 'whole-of-society and whole-of-country' approach including community mitigation measures.

\section{Outbreak preparedness}

The National Influenza Pandemic Preparedness Plan, which provides the basis for a coordinated and effective national response in the event of an influenza pandemic in the country, was activated during the initial stages (3). As the epidemic progressed, evolving epidemiological evidence and accrued new knowledge about the virus, underpinned decision making for a tailored response. A key element was to establish a robust and comprehensive surveillance system to capture all COVID-19 patients and their close contacts to contain the virus at the source.

\section{Strengthening the central response}

The central surveillance capacities have been strengthened by mobilizing the full capacity of the Epidemiology Unit for active surveillance. Standard Operational Procedures were developed, and an Emergency Operation Cell established at the Epidemiology Unit for active case search, contact tracing and follow-up for early case detection, and for guidance on assessing exposure risk and quarantining contacts. Communication systems were upgraded to enable a flawless information flow to facilitate networking with regional public health teams.

The National Avian Influenza Technical Committee was convened to deliberate on the way forward and to establish convergence plans for epidemic control. The National Advisory Committee on Communicable Diseases, which is a platform to obtain professional recommendations on policy directives pertinent to the current outbreak was convened for expert guidance.

\section{Strengthening the regional and institutional response}

The regional public health teams and institutional infection control teams play a pivotal role in outbreak response. Steps were taken to improve their response to COVID-19 coherent with national strategic directions. The regional epidemiologists (RE) were updated in early case detection, referral for isolation and treatment, tracing contacts, conducting community screening, and transporting specimens to laboratories. The infection control nursing officers from hospitals were trained on appropriate and effective infection control measures to be adopted in healthcare settings.

\section{Responding to the epidemic within the country}

The first confirmed COVID-19 patient in Sri Lanka was reported on 27 January through active case search, marking the start of the outbreak in Sri Lanka when a Chinese national touring the country tested positive for SARS-Co-V2. Since then, until the detection of the first Sri Lankan with COVID-19 on 11 March, the focus was on reducing the risk of importation of the disease. Continuous analysis of the global pandemic situation and appraisal of evidence from other countries on measures adopted for containment and mitigation helped to strategize the outbreak response.

\section{Community follow-up of returnees}

Prior to China closing its borders, there was potential to import cases from Wuhan, the epicentre of the virus and other Chinese cities with community spread. A mechanism was established, building on the communicable disease surveillance system of the country, to follow up returnees from high-risk areas in China. The Quarantine Unit and the Epidemiology Unit identified as the National Focal Points for International Health Regulations, took prompt action to implement all precautionary measures at ports of entry supported by the Disaster Preparedness and Response Division of the Ministry of Health. The returnees were registered at the ports of entry through the Health Declaration Forms. This information was then relayed to the medical officer of health $(\mathrm{MOH})$, where the returnees were residents through the RE. Public health inspectors (PHI) responsible for communicable disease surveillance at field level, with the guidance of MOHs, applied the concept of 
home quarantine and followed-up returnees for 14 days corresponding to the possible incubation period to enable early case detection. This process initiated for returnees from China was subsequently extended to returnees from South Korea and Italy on 24 and 29 February respectively; and later to returnees from other countries commensurate with the risk of introduction of the virus from these countries (4-5).

\section{Mandatory quarantining of returnees}

With an increasing number returning from countries with high disease transmission, the concept of cohort quarantine was adopted by the Ministry of Health. Accordingly, quarantine centres established by the military forces, initially to quarantine Sri Lankans evacuated from Wuhan, started admitting returnees from other high-risk countries from 18 March. Travellers and returnees who entered the country before this regulation was enacted resulted in the outbreak from 11 March 2020.

Guidance on the safe transportation of returnees to the designated centres and operational instructions to manage quarantine centres were shared with the tri forces designated to oversee the quarantine centres. Vigilant scrutiny of the global epidemiological situation facilitated the risk assessment and delineation of countries as high risk for disease transmission, enabling quarantining of returnees from these countries. As the epidemic progressed within the country, the scope of "cohort quarantine" was extended to 'hot spots' for disease transmission: local communities prone to disease amplification such as high-density urban areas. Under the guidance of the Epidemiology Unit, the quarantined individuals were appropriately tested, coordinated by the regional public health team and the quarantine centre staff, to prevent the transmission of infection back into the community.

\section{Surveillance of COVID-19 patients}

Epidemiological surveillance remains the cornerstone for the pandemic response in the country. Meticulous epidemiological surveillance has been carried out for each diagnosed case collaboratively with the regional public health staff.
The case definition for identifying COVID-19 patients has undergone a series of revisions in keeping with epidemiological trends, patient dynamics and symptom profile, demand driven scaling up of health sector capacities and WHO recommendations to capture all probable cases including epidemiologically linked asymptomatic cases thus preventing further spread.

Surveillance is multi-pronged with passive surveillance of suspected patients in hospitals, and active surveillance of close contacts of diagnosed patients, epidemiologically associated contacts and communities of patients with high potential transmissibility, healthcare staff, and suspected COVID-19 deaths.

Contact tracing is a core public health strategy adopted to interrupt chains of disease transmission in the country (5). This process is triggered by the reporting of positive cases by designated laboratories. A member of the contact tracing team of the Epidemiology Unit immediately makes initial contact with the patient over the phone. Travel histories, including travel abroad, is obtained to determine the source of infection and to classify cases as imported or locally acquired. Further, contacts are identified, and necessary technical advice given by the Epidemiology Unit. A list of close contacts and casual contacts prepared for each case is communicated with the respective RE. The RE immediately communicates with their provincial and district teams and together with relevant $\mathrm{MOH}$ and PHI conduct field-level investigations. They adopt a case-based intervention approach whereby each patient's house/contacts is visited to ascertain the exposure risk, institute quarantine measures, and direct for screening as required. The technical guidance of provincial/district consultant community physicians and REs ensure the quality of this process. The Epidemiology Unit together with the field health staff continue contact tracing until the chains of transmission are interrupted.

Surveillance data are disseminated daily through situation reports since the detection of the first imported case in Sri Lanka and through real-time updates of patients in the country on the 
Epidemiology Unit website. Surveillance data is useful in risk communication to the public to understand the situation of the country and to sustain trust in the government's response to COVID-19. Surveillance information has also been the basis to advocate for an appropriate and proportionate response to the risk imposed by the disease minimizing negative economic and societal impacts.

Development of guidelines, circulars, and protocols

One of the key priorities has been to streamline the response to COVID-19 by establishing protocols and formulating guidelines with the collaboration of relevant other experts in different disciplines. Interim guidance for Sri Lankan flights to and from China and advice for Sri Lankan Students/Nationals and other foreign nationals returning from high-risk areas of on-going COVID-19 transmission was developed during the early phase of the response when intercountry travel was still operational (6-7). A guideline for self-quarantining at home or quarantining in nonhealthcare settings was issued to contain any risk of spread of infection from those returning from abroad (8).

Being cognizant of the shortages in personal protective equipment (PPE) around the globe and the need to protect our healthcare workers without compromising their ability to respond to the epidemic, interim guidance was issued on the rational use of PPE in hospitals in collaboration with consultant microbiologists (9).

Scaling-up laboratory facilities has been of paramount importance in responding to COVID-19, for which the Epidemiology Unit furnished necessary epidemiological evidence to guide decisions. The National Testing Criteria for COVID-19 has been revised in response to the epidemiological demand and surveillance goals in expanding case detection. The COVID-19 laboratory test strategy in Sri Lanka was developed to rationalize testing in different risk scenarios while optimizing resource use (10).
Inter-sectoral collaboration for a unified response

The pandemic has called for an unprecedented coordination and a unified national COVID-19 response from all relevant health and non-health parties and the Epidemiology Unit has cooperated and advocated based on epidemiologic intelligence for an integrated response. Real-time epidemiological data have been provided to guide decisions including population-level movement restrictions.

The Epidemiology Unit continues to monitor, evaluate, learn, and advocate as the epidemic progresses to prevent it becoming a crisis based on surveillance under the authority of the Director General of Health Services. This would eventually help to mitigate devastating impacts on the socioeconomic profile of the country.

\section{Sustaining the momentum}

COVID-19 is the defining global health crisis of our times. Countries have risen to the challenge adapting a common set of suppression and mitigation measures aligned with the socio-political and economic fabric of each country and the capacity of their health systems. Sri Lanka has thus far managed to keep the growth of the epidemic at a slow pace and has economized the opportunity to improve the resilience of the health system and the people as a whole. Sri Lanka has a strategy of admitting all patients, regardless of symptom manifestation, unlike in countries where asymptomatic and mild cases are managed at home.

The future is challenging. With the relaxation of population movement restrictions and opening of ports of entry there is a risk for another surge in cases unless stringent surveillance is maintained to promptly detect cases along with population and individual level interventions such as maintaining physical distancing, respiratory and hand hygiene. To this end, the Epidemiology Unit is committed to continue to fulfil its role as the technical lead for surveillance in collaboration with the regional health staff and other stakeholders whose contribution to date has been exemplary and encouraging. 


\section{Author Declaration}

Acknowledgements: The authors gratefully acknowledge the contribution made by the staff of the Epidemiology Unit, and the national/provincial /regional preventive and curative health staff towards COVID 19 surveillance.

Author contributions: NW and $\mathrm{CH}$ drafted and revised the manuscript for final approval; DG reviewed and edited the manuscript; TR, CJ, MK, SW, AP, TH, SS, PP and SG reviewed and commented on the manuscript. All authors read and approved the final manuscript.

\section{References}

1. World Health Organization. Novel Coronavirus (2019-nCoV). Situation Report 1, 20 January 2020, World Health Organization. Available from: https:// www.who.int/docs/default-source/ coronaviruse/situation-reports/20200121-sitrep1-2019-ncov.pdf?sfvrsn= 20a99c10_4. Accessed 15 May 2020.

2. World Health Organization. Statement on the second meeting of the International Health Regulations. Emergency Committee regarding the outbreak of novel coronavirus (2019-nCoV). World Health Organization 2020. Available from: https://www.who.i nt/news-room/detail/30-012020-statement-on-the-second-meeting-of-theinternational-health-regulations-(2005)emergency-committee-regarding-the-outbreakof-novel-coronavirus-(2019-ncov). Accessed 14 May 2020.

3. Epidemiology Unit. National Influenza Pandemic Preparedness Plan- 2012. Epidemiology Unit, Ministry of Health. Available from: https://www.epid.gov.lk/web/images/pdf/ Influenza/completed_nipp_2013-03-20.pdf Accessed 14May 2020.

4. Ministry of Health and Indigenous Medical Services. Follow up of Sri Lankan Nationals and other foreign Nationals returning from high risk areas for ongoing Coronavirus (COVID-19) transmission. Ministry of Health, 2020. Available from: http:// www.e pid.gov.lk/web/images/pdf/ Circulars/Corona_virus/Followup-of-Sri-lankannationals-and-other-foreign-nationals.pdf. Accessed 12 May 2020.

5. World Health Organization. Contact tracing in the context of COVID-19. World Health Organization, 2020. Available from: https:// www.who.int/publications-detail/contacttracing-in-the-context-of-covid-19. Accessed 12 May 2020.

6. Epidemiology Unit. Interim Guidance for Sri Lankan flights to and from China. Epidemiology Unit, Ministry of Health. Available from: http://www.epid. gov.lk /web/images/pdf/ Circulars/Corona_virus/instructions to flight from china_10.2. 2020_final1.pdf. Accessed 12 May 2020.

7. Epidemiology Unit. Advices for Sri Lankan students/nationals and other foreign nationals returning from high risk areas of on-going coronavirus transmission. Epidemiology Unit, Ministry of Health. Available from: http://www.epid.gov.lk /web/images/pdf/ Circulars/Corona_virus/advices for Sri Lankan students.pdf. Accessed 14 May 2020.

8. Epidemiology Unit. Guideline for the Home quarantinel quarantine in non-health care settings. Epidemiology Unit, Ministry of Health. Available from: http://www.epid.gov .lk/web/images/pdf/Circulars/Corona_virus/guid elines-ofhome-quarantine.pdf. Accessed 12 May 2020.

9. Epidemiology Unit. Guidance on the rational use of personal protective equipment (PPE) in hospitals in the context of COVID-19 disease. Epidemiology Unit, Ministry of Health. Available from: http://www.epid .gov.lk/web/images/pdf/ Circulars/Corona_virus/ guidance-on-therational-use-of-personal-protective-equipment. pdf. Accessed 12May 2020.

10. Epidemiology Unit. COVID 19 laboratory testing strategy in Sri Lanka. Epidemiology Unit, Ministry of Health. Available from: http:// www.epid.gov.lk/web/images/pdf/Circulars/Cor ona_virus/final_draft_of_testing_strategy.pdf. Accessed 12May 2020. 